\title{
Optimal cytoreductive surgery for underlying ovarian cancer associated with deep venous thrombosis without placement of inferior vena cava filter: A case report and literature review
}

\author{
HONGWEI SHEN* ${ }^{*}$ JIANHONG SHANG* ${ }^{*}$ GANG NIU, JUN LIU, ZESHAN YOU and SHANYANG HE \\ Department of Obstetrics and Gynecology, The First Affiliated Hospital, Sun Yat-Sen University, \\ Guangzhou, Guangdong 510080, P.R. China
}

Received November 6, 2014; Accepted May 27, 2015

DOI: $10.3892 / 01.2015 .3497$

\begin{abstract}
Ovarian cancer associated with deep venous thrombosis (DVT) is an uncommon, potentially life-threatening condition. The primary therapeutic strategy for the treatment of this condition is up-front primary cytoreductive surgery, with placement of an inferior vena cava (IVC) filter prior to surgery to prevent fatal pulmonary embolism. The present study describes the case of a 49-year-old female, who presented with DVT unresponsive to anticoagulant therapy in the lower extremities prior to the diagnosis of ovarian cancer. During the search for the underlying malignancy, transvaginal sonography (TVS) revealed a cystic solid mass in the pelvic cavity. Subsequently, the patient underwent up-front primary cytoreductive surgery without placement of a preoperative IVC filter, followed by six cycles of chemotherapy. The patient was diagnosed with ovarian clear cell adenocarcinoma stage IIIC, complicated by DVT, and had survived $>3$ years without relapse at the time of completion of the present study. The successful outcome of the present case demonstrated that occult primary cancer should be suspected in patients with DVT unresponsive to anticoagulant therapy. The present study also indicated that up-front primary cytoreductive surgery without placement of an IVC filter represents an effective potential strategy for the treatment of advanced ovarian cancer associated with DVT, as the thrombus strongly adheres to the vessel wall following organization.
\end{abstract}

Correspondence to: Dr Shanyang He, Department of Obstetrics and Gynecology, The First Affiliated Hospital, Sun Yat-Sen University, 58 Zhongshan $2^{\text {nd }}$ Road, Guangzhou, Guangdong 510080, P.R. China E-mail: hsy5g777@sina.com

*Contributed equally

Key words: deep venous thrombosis, inferior vena cava filter, ovarian cancer, up-front primary cytoreductive surgery

\section{Introduction}

Epithelial ovarian cancer results in mortality more frequently than any other gynecologic malignancy, as the majority of patients present with advanced stage disease (1). Up-front primary cytoreductive surgery followed by chemotherapy is able to improve overall survival, and remains the standard treatment for the majority of patients with resectable advanced ovarian cancer (2). Compared with other cancers, ovarian cancer, and particularly clear cell carcinoma, is associated with a high incidence of deep venous thrombosis (DVT), which may affect surgical outcomes. The overall incidence of symptomatic DVT in ovarian cancer is $9.7-16.6 \%(3,4)$. However, the detection of DVT prior to the diagnosis of ovarian cancer is rare, with an estimated incidence of $3.2 \%$ (3). Up-front primary cytoreductive surgery remains a significant clinical challenge for patients with DVT.

Inferior vena cava (IVC) filters are typically placed in the perioperative period of primary cytoreductive surgery to reduce the risk of DVT-associated pulmonary embolism (PE) (5-7). Although IVC filters effectively prevent PE, they are do not improve survival rates in patients with ovarian cancer undergoing primary cytoreductive surgery, due to their significant associated complications $(8,9)$ and the high risk of hematogenous distant metastasis in this population (10). Therefore, the use of IVC filters in combination with up-front primary cytoreductive surgery for ovarian cancer in patients with DVT is controversial.

The current study presents the rare case of a patient with DVT in the two lower extremities, identified prior to the diagnosis of clear cell ovarian cancer. The patient underwent optimal primary cytoreductive surgery within 1 month of unsuccessful anticoagulant therapy, without placement of a preoperative IVC filter. The patient's surgical outcome was good, with resolution of lower limb swelling and without PE. The patient was followed-up for $>3$ years, during which time serum tumor marker assays and transvaginal sonography (TVS) identified no evidence of tumor recurrence. The present case provides significant information regarding the potential management of ovarian cancer in patients with DVT. Other published case reports of ovarian cancer associated with DVT in the literature were also evaluated. 

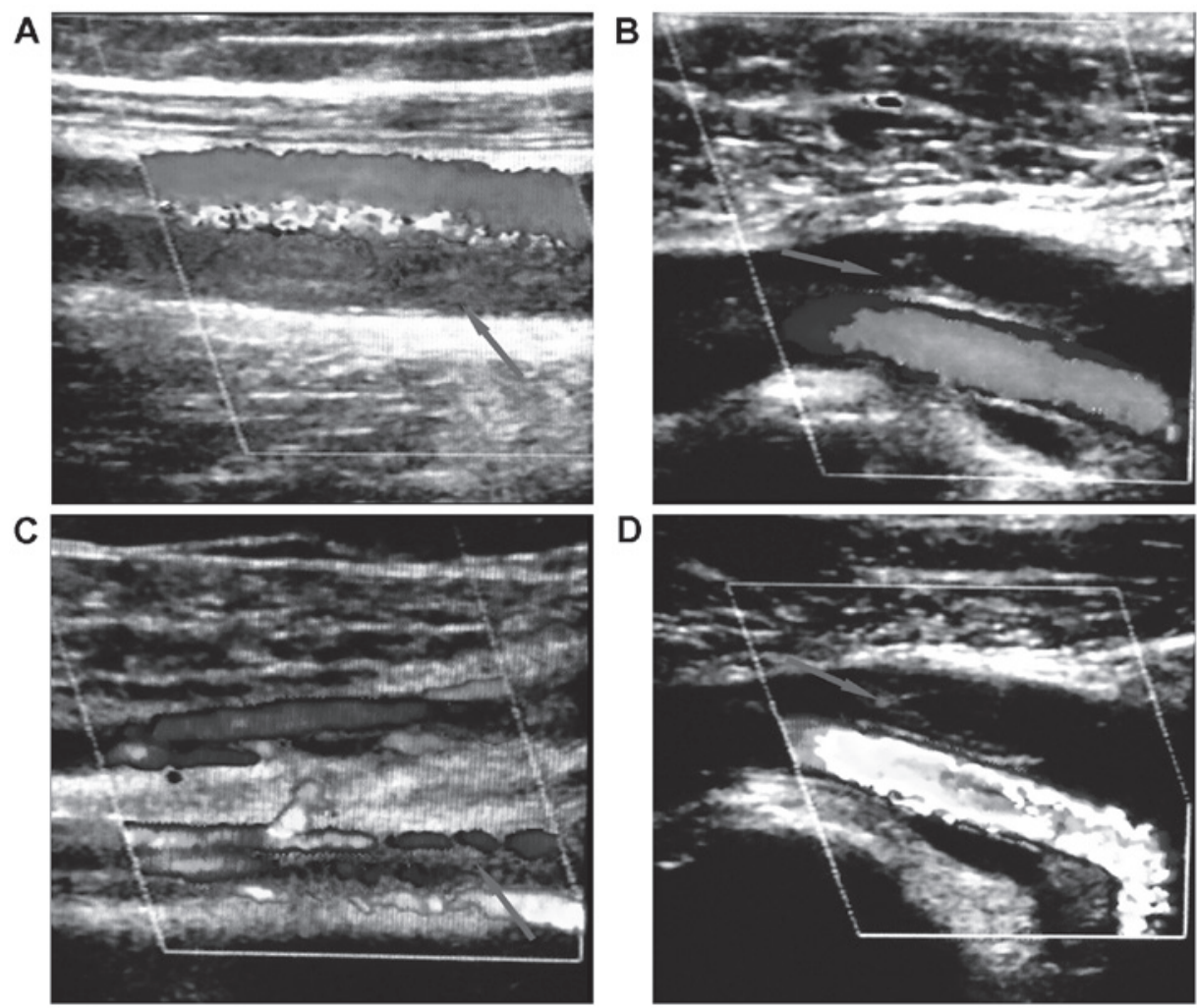

Figure 1. Color Doppler flow images revealing DVT involving the lower extremities. (A) DVT in the superficial femoral vein of the right leg. (B) DVT in the popliteal vein of the right leg. (C) DVT in the fibular vein of the right leg. (D) DVT in the popliteal vein of the left leg detected following 2 weeks of anticoagulant therapy. DVT, deep venous thrombosis.

\section{Case report}

Written consent from the patient for the use of clinical materials for research purposes and approval from the Institutional Ethics Board of the First Affiliated Hospital of Sun Yat-Sen University (Guangzhou, China) were obtained.

A 49-year-old female [body mass index (BMI), $24.0 \mathrm{~kg} / \mathrm{m}^{2}$ ] presented to the Vascular Surgery Department at the First Affiliated Hospital, Sun Yat-Sen University with reddish swelling and pain in the right leg, which had persisted for 3 days. The difference in circumference between the patient's lower limbs was $2.3 \mathrm{~cm}$ (left, $34.5 \mathrm{~cm}$; right, $36.8 \mathrm{~cm}$ ). Color Doppler flow imaging of the right lower extremity identified multiple DVTs in the superficial femoral, popliteal and fibular veins (Fig. 1A-C). Coagulation function tests were performed, including platelet count $\left(219 \times 10^{9} / 1\right)$, D-dimer $[18,190.00 \mu \mathrm{g} / 1$, increased compared with normal values (increased)], prothrombin time (17.8 sec, increased), activated partial thromboplastin time $(30.6 \mathrm{sec})$, thrombin time $(17.2 \mathrm{sec})$, fibrinogen $(2.00 \mathrm{~g} / \mathrm{l})$ and international normalized ratio (1.6, increased). The patient had no history of trauma, surgery, hormone therapy or smoking, and was also single and without a sexual history. The patient's family history was negative for clotting disorders. The patient underwent low molecular weight heparin anticoagulation therapy. However, the patient's symptoms did not improve following 2 weeks of continuous therapy, and the popliteal vein in the lower left extremity developed a thrombus with $80 \%$ stenosis (Fig. 1D). Occult malignancy was suspected and therefore, the expression of 10 serum tumor markers was measured: Human epididymis protein (168.7 pmol/1, increased), cancer antigen (CA) 153 (3,000 U/ml, increased), CA72-4 (15.09 U/ml, increased), CA125 (541.5 U/ml, increased), CA199 (111.4 U/ml, increased), carcinoembryonic antigen $(0.619 \mathrm{ng} / \mathrm{ml})$, neuron-specific enolase (24.78 ng/ml, increased), human chorionic gonadotropin- $\beta(0.100 \mathrm{mIU} / \mathrm{ml})$, squamous cell carcinoma antigen $(0.7 \mathrm{ng} / \mathrm{ml})$ and $\alpha$-fetoprotein $(2.84 \mathrm{ng} / \mathrm{ml})$. TVS revealed a cystic solid mass measuring $16.0 \times 12.8 \mathrm{~cm}$ in the pelvic cavity (Fig. 2A). The patient was admitted to the Obstetrics and Gynecology Department for further treatment. Computed tomography demonstrated right ovarian carcinoma in the pelvic cavity, with cystic and solid portions (Fig. 2B). The patient required surgery, but refused placement of a perioperative IVC filter due to insufficient funds. Exploratory laparotomy confirmed the presence of a right ovarian mass with a maximum diameter of $16.0 \mathrm{~cm}$ and $500 \mathrm{ml}$ ascitic fluid in the abdominal cavity (Fig. 2C and D). A right oophorectomy was performed and an intraoperative frozen section was obtained for the confirmation of diagnosis. The solid and cystic mass exhibited an uneven surface with excrescences, and the section was comprised of brittle tissue. Following $30 \mathrm{~min}$ of evaluation, pathology confirmed ovarian epithelial malignancy. Aggressive total hysterectomy, right salpingectomy, left salpingo-oophorectomy, omentectomy, pelvic and para-aortic lymph node dissection and appendectomy were performed. The liver, pancreas, gall bladder, stomach, and small and large bowel remained normal. No intraoperative peritoneal deposits or areas suspicious for malignancy were found. Intraoperative bleeding was $200 \mathrm{ml}$. Heparin anticoagulation therapy was stopped $12 \mathrm{~h}$ prior to surgery, and resumed $24 \mathrm{~h}$ post surgery. 

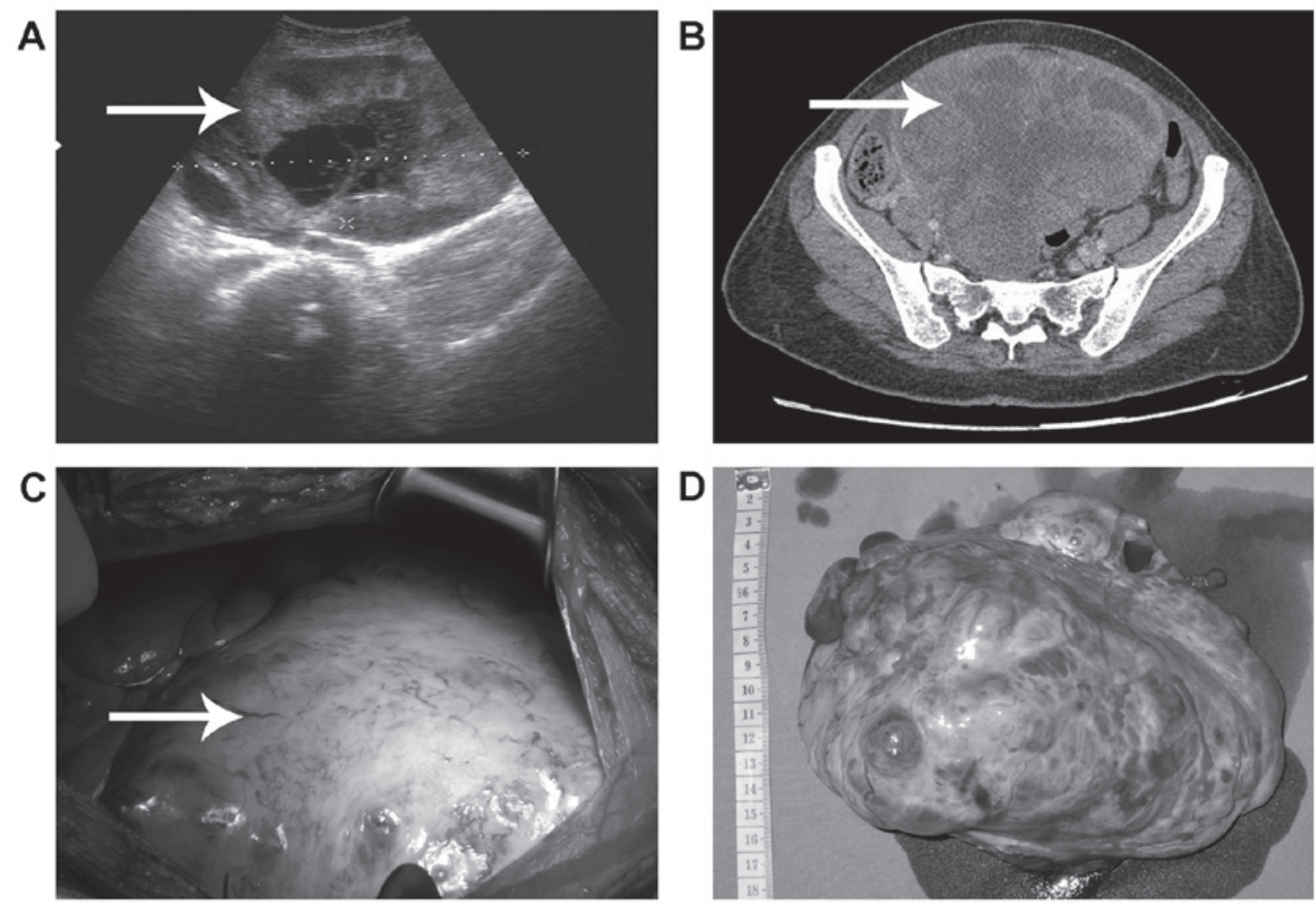

Figure 2. Giant mass identified in the pelvic cavity. (A) Transvaginal sonography identified a cystic solid mass in the pelvic cavity. (B) Computed tomography revealed right ovarian carcinoma with cystic and solid portions. (C) Exploratory laparotomy revealed a giant ovarian carcinoma in the pelvic cavity. (D) The solid cystic mass had an uneven surface with excrescences and a maximum diameter of $16.0 \mathrm{~cm}$.
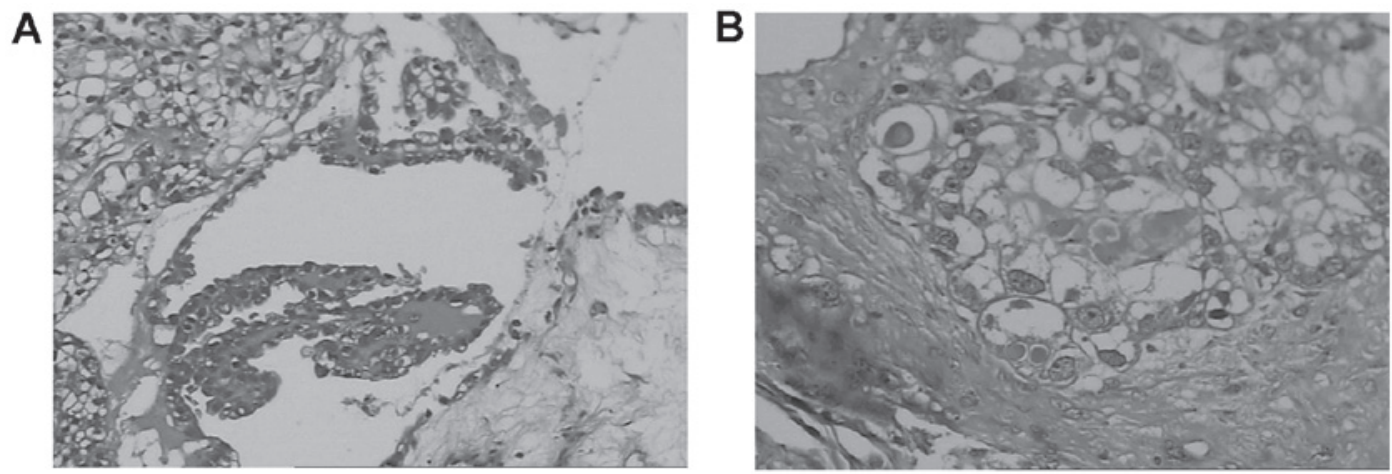

Figure 3. Histological analysis indicates clear cell adenocarcinoma, grade IIIC. (A) Cystic and papillary areas were visible with typical hobnailing. H\&E staining; magnification, x400; (B) Eosinophilic bodies lie in the cytoplasm of clear cytoplasmic cells. H\&E staining; magnification, x400. H\&E, hematoxylin and eosin.

On postoperative day (POD) 1, the patient's DVT symptoms were significantly relieved, with a decreased difference in lower limb circumference (left, $30.5 \mathrm{~cm}$; right, $31.5 \mathrm{~cm}$ ). In addition the plasma level of D-dimer had been reduced to $4,440.00 \mu \mathrm{g} / \mathrm{l}$. Histology of the tumor confirmed ovarian clear cell adenocarcinoma (Fig. 3A and B) with left iliac and obturator lymph node metastasis. The patient's uterus, right adnexa, and omentum majus were negative for metastasis. The cancer stage was IIIC. The patient received six cycles of carboplatin (AUC) $=6$ and docetaxel $\left(175 \mathrm{mg} / \mathrm{m}^{2}\right)$ every 4 weeks following surgery. Serum tumor markers were measured following the first cycle of chemotherapy. Serum CA153 had reduced from $3,000 \mathrm{U} / \mathrm{ml}$ to $185.20 \mathrm{U} / \mathrm{ml}$, while CA125 had decreased from $541.5 \mathrm{U} / \mathrm{ml}$ to $73.70 \mathrm{U} / \mathrm{ml}$. CA199 and carcinoembryonic antigen levels were normal. Warfarin oral anticoagulation was continued and the international normalized ratio was maintained in the treatment range of 2.0-3.0. All serum tumor marker levels and plasma D-dimer levels returned to normal following the second cycle of chemotherapy. The patient was followed-up for $>3$ years, during which time serum tumor marker assays and TVS indicated no evidence of tumor recurrence. The successful outcome of the present case suggests that ovarian cancer with DVT may be treated with up-front primary cytoreductive surgery within 1 month of anticoagulant therapy, without placement of an IVC filter.

\section{Discussion}

Venous thromboembolism (VTE), including DVT and PE, is increasingly recognized as a common complication amongst patients with malignant disease, and is associated with increased mortality, recurrence and compromised quality of 
life (11-13). Numerous studies have confirmed this association between malignancy and VTE $(12,14,15)$. The overall risk for VTE is seven-fold greater in patients with malignancy, compared with that of individuals without malignancy, particularly in the first few months following diagnosis, and in the presence of distant metastases (12). Among solid tumors, ovarian cancer has one of the highest incidence rates of VTE, an effect which adversely impacts patient survival $(16,17)$. The overall incidence of symptomatic DVT among patients with ovarian cancer is reported to be $9.7-16.6 \%$, with $3.2 \%$ of cases detected prior to the diagnosis of ovarian cancer $(3,4)$. In the present case, DVT was unresponsive to anticoagulant therapy, suggesting the prescence of an underlying malignancy. Optimal cytoreductive surgery without placement of a preoperative IVC filter was performed, and ovarian cancer was confirmed. This case suggests that clinicians should suspect underlying malignancy when DVT is unresponsive to anticoagulant therapy.

Factors contributing to the risk of DVT in cancer patients include clear cell subtype, high grade and stage of cancer, high CA125 (>500 IU/ml), giant pelvic mass, history of DVT, ongoing chemotherapy, lack of surgery, presence of residual tumor and $\mathrm{BMI} \geq 30$ (4). Clear cell carcinomas are associated with the highest DVT incidence (27.3\%), compared with that of $6.8 \%$ among patients with other types of epithelial ovarian cancer (18). The present patient exhibited several risk factors for DVT, including high CA125 level, advanced stage, giant pelvic tumor and clear cell adenocarcinoma subtype; although had no history of DVT and a normal BMI. The symptoms of DVT were significantly relieved by POD 1 , with a decreased difference in circumference between the lower limbs, resulting from decreased pelvic pressure. Tumor-associated obstruction of venous flow may have a more prominant role in gynecologic cancer patients than hypercoagulability activation by tumor cells (19). Clinicians should be aware of the risk factors for DVT so that appropriate perioperative prophylactic measures, including anticoagulation therapy, may be effectively used. Furthermore, in this case, primary surgery was effective for a resectable giant pelvic mass with DVT.

However, the management of ovarian cancer associated with DVT remains challenging. There are currently three approaches to the management of such cases: i) Up-front primary cytoreductive surgery with placement of an IVC filter prior to surgery to prevent PE (20); ii) neoadjuvant chemotherapy followed by interval debulking surgery for huge tumors that cannot be treated with optimal primary cytoreductive surgery (20); and iii) initial less-invasive diagnostic laparoscopy to remove cancer tissue, followed by three cycles of chemotherapy and complete staging surgery, an approach restricted to patients with small ovarian tumors (21). Up-front primary cytoreductive surgery with preoperative IVC filter placement provides a histopathological diagnosis, removes as much cancer tissue as possible, establishes the International Federation of Gynecology and Obstetrics stage (2), aids complete resection of macroscopic disease (22) and improves overall survival $(20,23)$, compared with the effects of neoadjuvant chemotherapy for resectable advanced ovarian cancer. However, numerous complications have been reported to be associated with IVC filters, including IVC penetration, filter embolization and movement, filter fracture, recurrent
PE and DVT $(8,9)$. Furthermore, perioperative placement of an IVC filter in patients with ovarian cancer undergoing primary cytoreductive surgery may significantly decrease survival time and elevate the risk of DVT and hematogenous distant metastasis (10). In addition, these patients should be monitored closely and the filter requires removal once anticoagulation is no longer contraindicated (24). The use of IVC filters prior to primary cytoreductive surgery therefore remains controversial.

The present study describes the case of a huge ovarian tumor with DVT, treated within 1 month of anticoagulant therapy with optimal primary cytoreductive surgery without placement of an IVC filter. A preoperative IVC filter was not placed in this case as the patient did not suffer from clinically evident PE and the chronic venous thrombi were adhered to the vessel wall and did not involve the pelvic veins, lowering the risk of PE (25). In addition, the patient and family refused placement of an IVC filter due to a lack of funds. Based on these circumstances, optimal cytoreductive surgery was performed without placement of a preoperative IVC filter. This case differed from those of previous studies $(20,21)$. The patient was followed up for $>3$ years, during which time serum tumor marker assays and TVS scans identified no evidence of tumor recurrence. Although no adverse events have previously been reported following surgery without placement of an IVC, the number of cases is too low to draw general conclusions. The present case is therefore a useful contribution to enhancing understanding of the treatment of ovarian cancer with DVT.

In conclusion, occult cancer should be suspected in patients with DVT unresponsive to anticoagulant therapy, and optimal cytoreductive surgery without placement of a preoperative IVC filter is a feasible treatment strategy for advanced ovarian cancer with DVT, when the thrombus strongly adheres to the vessel wall organization.

\section{Acknowledgements}

The present study was supported by the Science and Technology Development of Guangdong, China (nos. 2011B031800100 and 2011B031800025) and the Nature Science Committee of Guangdong, China (nos. S2013010015599 and S2012010008270).

\section{References}

1. Jayson GC, Kohn EC, Kitchener HC and Ledermann JA: Ovarian cancer. Lancet 384: 1376-1388, 2014.

2. Morgan RJ Jr, Alvarez RD, Armstrong DK, Burger RA, Castells M, Chen LM, Copeland L, Crispens MA, Gershenson D, Gray H, et al; National Comprehensive Cancer Network: Ovarian cancer, version 3.2012. J Natl Compr Canc Netw 10: 1339-1349, 2012.

3. Tateo S, Mereu L, Salamano S, Klersy C, Barone M, Spyropoulos AC and Piovella F: Ovarian cancer and venous thromboembolic risk. Gynecol Oncol 99: 119-125, 2005.

4. Abu Saadeh F, Norris L, O'Toole S and Gleeson N: Venous thromboembolism in ovarian cancer: Incidence, risk factors and impact on survival. Eur J Obstet Gynecol Reprod Biol 170: 214-218, 2013.

5. Dewdney SB, Benn T, Rimel BJ, Gao F, Saad N, Vedantham S, Mutch DG and Zighelboim I: Inferior vena cava filter placement in the gynecologic oncology patient: A 15-year institutional experience. Gynecol Oncol 121: 344-346, 2011. 
6. Sing RF and Fischer PE: Inferior vena cava filters: Indications and management. Curr Opin Cardiol 28: 625-631, 2013.

7. Duffett LD, Gándara E, Cheung A, Bose G, Forster AJ and Wells PS: Outcomes of patients requiring insertion of an inferior vena cava filter: A retrospective observational study. Blood Coagul Fibrinolysis 25: 266-271, 2014.

8. Sella DM and Oldenburg WA: Complications of inferior vena cava filters. Semin Vasc Surg 26: 23-28, 2013.

9. Lee JK, So YH, Choi YH, Park SS, Heo EY, Kim DK and Chung HS: Clinical course and predictive factors for complication of inferior vena cava filters. Thromb Res 133: 538-543, 2014.

10. Matsuo K, Carter CM, Ahn EH, Prather CP, Eno ML, Im DD and Rosenshein NB: Inferior vena cava filter placement and risk of hematogenous distant metastasis in ovarian cancer. Am J Clin Oncol 36: 362-367, 2013

11. Noble S and Pasi J: Epidemiology and pathophysiology of cancer-associated thrombosis. Br J Cancer 102 (Suppl 1): S2-S9, 2010.

12. Blom JW, Doggen CJM, Osanto S and Rosendaal FR: Malignancies, prothrombotic mutations, and the risk of venous thrombosis. JAMA 293: 715-722, 2005.

13. Arora M and Wun T: Adverse impact of venous thromboembolism on patients with cancer. Semin Thromb Hemost 40: 313-318, 2014

14. Bierry G, Holl N, Kellner F, Riehm S, Roedlich MN, Greget M and Veillon F: Venous thromboembolism and occult malignancy: Simultaneous detection during pulmonary CT angiography with CT venography. AJR Am J Roentgenol 191: 885-9, 2008.

15. Murchison JT, Wylie L and Stockton DL: Excess risk of cancer in patients with primary venous thromboembolism: A national, population-based cohort study. Br J Cancer 91: 92-95, 2004.

16. Metcalf RL, Fry DJ, Swindell R, McGurk A, Clamp AR, Jayson GC and Hasan J: Thrombosis in ovarian cancer: A case control study. Br J Cancer 110: 1118-1124, 2014.

17. Gunderson CC, Thomas ED, Slaughter KN, Farrell R, Ding K, Farris RE, Lauer JK, Perry LJ, McMeekin DS and Moore KN: The survival detriment of venous thromboembolism with epithelial ovarian cancer. Gynecol Oncol 134: 73-77, 2014.
18. Matsuura Y, Robertson G, Marsden DE, Kim SN, Gebski V and Hacker NF: Thromboembolic complications in patients with clear cell carcinoma of the ovary. Gynecol Oncol 104: 406-410, 2007.

19. Abu Saadeh F, Norris L, O'Toole S, Mohamed BM, Langhe R, O'Leary J and Gleeson N: Tumour expression of tissue factor and tissue factor pathway inhibitor in ovarian cancer - relationship with venous thrombosis risk. Thromb Res 132: 627-634, 2013.

20. Hurtt CC, Cliby WA, Weaver AL, McGree M, Martin J and Bakkum-Gamez JN: Management of epithelial ovarian cancer in the setting of concomitant venous thromboembolism. Obstet Gynecol 123 (Suppl 1): 183S-184S, 2014.

21. Ha JE, Lee YS, Lee HN and Park EK: Diagnostic laparoscopy of patient with deep vein thrombosis before diagnosis of ovarian cancer: A case report. Cancer Res Treat 42: 48-52, 2010

22. Vergote I, Tropé CG, Amant F, Kristensen GB, Ehlen T, Johnson N, Verheijen RH, van der Burg ME, Lacave AJ, Panici PB, et al; European Organization for Research and Treatment of Cancer-Gynaecological Cancer Group; NCIC Clinical Trials Group: Neoadjuvant chemotherapy or primary surgery in stage IIIC or IV ovarian cancer. N Engl J Med 363: 943-953, 2010.

23. van Meurs HS, Tajik P, Hof MH, Vergote I, Kenter GG, Mol BW, Buist MR and Bossuyt PM: Which patients benefit most from primary surgery or neoadjuvant chemotherapy in stage IIIC or IV ovarian cancer? An exploratory analysis of the European Organisation for Research and Treatment of Cancer 55971 randomised trial. Eur J Cancer 49: 3191-3201, 2013.

24. Al-Hakim R, Kee ST, Olinger K, Lee EW, Moriarty JM and McWilliams JP; AI-Hakim R: Inferior vena cava filter retrieval: Effectiveness and complications of routine and advanced techniques. J Vasc Interv Radiol 25: 933-939, 2014.

25. Köhn H, König B and Mostbeck A: Incidence and clinical feature of pulmonary embolism in patients with deep vein thrombosis: A prospective study. Eur J Nucl Med 13 (Suppl): S11-S15, 1987. 